\title{
Global emergence of a multi-drug resistant fungal pathogen, Candida auris
}

\author{
Erika Britz ${ }^{\mathrm{a}}$ and Nelesh P Govender ${ }^{\mathrm{ab} *}$ \\ ${ }^{a}$ National Institute for Communicable Diseases [Centre for Opportunistic, Tropical and Hospital Infections], Johannesburg, South Africa \\ ${ }^{b}$ Division of Medical Microbiology, Faculty of Health Sciences, University of Cape Town, South Africa \\ *Corresponding author. email: Neleshg@nicd.ac.za
}

\begin{abstract}
An emerging fungal "superbug"
A multi-drug resistant fungus, Candida auris, has recently emerged on several continents as a cause of invasive human infections. First described in Japan in 2009, invasive infection with C. auris is associated with a crude mortality ranging from $33 \%$ to $72 \% .^{2-5} \mathrm{C}$. auris has been reported to cause a wide range of clinical manifestations, including fungaemia, wound sepsis and otitis, and cases have since been described in South Korea, ${ }^{3,6}$ India, ${ }^{4,5}$ Kuwait ${ }^{7}$ and South Africa. ${ }^{8}$ This pathogen has also been identified more recently in Colombia, Venezuela, Pakistan and the United Kingdom. ${ }^{9} 10$ Molecular studies documented recent, nearsimultaneous and independent emergence of $C$. auris on three different continents. ${ }^{2}$ Of most concern, almost all tested isolates are resistant to fluconazole, an important first-line antifungal agent, and in a recent global study, more than $40 \%$ of isolates were resistant to two or more major classes of antifungals. $2,4,5,7,8,11$
\end{abstract}

\section{Epidemiology}

Infections with $C$. auris have been largely acquired in hospital settings. A recent collaborative study, describing case patients from four countries, found a median time of 19 days (IQR: 9-36) from admission to diagnosis of $C$. auris infection, with diabetes mellitus, recent surgery and the presence of a central venous catheter (CVC) or urinary catheter in situ as common underlying factors. ${ }^{2}$ Chronic kidney disease, haematological malignancies, broad-spectrum antimicrobial agents and admission to an intensive care unit have also been documented as risk factors for C. auris infection..$^{5,6}$ In South Africa, urine, CVC tips and blood cultures are among the most common specimens from which $C$. auris has been isolated (Magobo et al., unpublished data).

Healthcare-associated outbreaks of $C$. auris have been reported in at least two countries. ${ }^{2,9}$ Although the mode of transmission in these outbreaks is not established, researchers have hypothesised that $C$. auris might contaminate the immediate environment of infected or colonised patients with onward transmission from the hands of healthcare workers or on fomites. ${ }^{9}$

C. auris has rapidly emerged as a common cause of candidaemia and other infections in hospitals in Gauteng province, with several hundred laboratory-confirmed cases documented since 2013 (Magobo et al., unpublished data). Sporadic cases have been detected in other provinces. Molecular epidemiology work supports nosocomial transmission of $C$. auris in South African hospitals. For example, multi-locus sequence typing (MLST) analysis of $79 \mathrm{C}$. auris isolates from patients admitted to several public-sector and private-sector hospitals in Gauteng province, confirmed genetic relatedness of isolates. ${ }^{11}$ Typing of isolates by amplified fragment length polymorphism (AFLP), MLST and matrix-assisted laser desorption ionisation-time of flight mass spectrometry has shown clonality of $C$. auris isolates in India, ${ }^{4,5}$ South Africa and Venezuela. ${ }^{10}$ More recently, whole genome sequence analysis of $C$. auris isolates from India, Pakistan, Venezuela and South Africa showed that strains from each continent clustered into distinct groups with low genetic diversity among isolates within each group. ${ }^{2}$ This strongly suggested independent emergence on different continents, rather than spread from one region to another.

\section{Laboratory and antifungal susceptibility testing}

C. auris is frequently misidentified in the routine diagnostic laboratory using commercially-available biochemical methods. Phenotypically, C. auris is difficult to distinguish from other pathogenic Candida species; it is a germ tube test-negative budding yeast, forms cream-coloured yeast-like colonies on nonselective agar and pale purple or pink colonies on chromogenic agar. ${ }^{12}$ The VITEK-2 YST and VITEK Mass Spectrometry (MS) systems (bioMérieux, France) currently misidentify this pathogen as Candida haemulonii, API systems (bioMérieux, France) as Rhodotorula glutinis, and Auxacolor (Bio-Rad, USA) as Saccharomyces cerevisiae. It has also been misidentified as Candida sake and Candida famata. Identification of $C$. auris can currently be confirmed using the MALDI Biotyper system (Bruker Corporation, USA) or molecular methods. A software update should be immediately available for the VITEK MS system, which will enable accurate identification of $C$. auris, and an update to the VITEK-2 YST system is planned for early 2017 (Alfie Clarke, bioMérieux, South Africa. personal communication. A formal signed letter from Alfie Clarke (microbiology product manager) to all bioMerieux customers (in response to a letter from the South African Society for Clinical Microbiology) was dated 27 July 2016).

Both species-level identification and antifungal susceptibility testing of invasive isolates is essential to guide patient management. However, there are no agreed-upon interpretive breakpoints for $C$. auris and any antifungal agent. The United States Centers for Disease Control and Prevention has applied conservative breakpoints, developed for other Candida species, to C. auris for epidemiological purposes; however, these may not necessarily be clinically relevant at an individual patient level. ${ }^{9}$ Interestingly, whole genome sequencing has identified three different amino acid substitutions in the ERG11 gene of $C$. auris, each mutation associated with isolates from a different continent, implying that resistance to fluconazole might be acquired rather than intrinsic. ${ }^{2}$

\section{Treatment and infection prevention and control measures}

Public Health, England, currently recommends an echinocandin (i.e. caspofungin, micafungin or anidulafungin) as first-line therapy for $C$. auris infections, pending antifungal susceptibility testing..$^{12}$ As echinocandins may not be available in the South African public sector, amphotericin $B$ is a reasonable alternative. No amphotericin B resistance has been confirmed in South Africa to date (Govender et al., unpublished data). 
Currently-recommended IPC measures include isolation, or "cohorting", of infected patients, standard contact (barrier) precautions and environmental decontamination, where feasible. The environment of an infected patient is recommended to be thoroughly cleaned every day using a chlorine-releasing agent, ${ }^{12}$ and hydrogen peroxide vapour, ${ }^{12}$ or an EPA-registered hospital grade disinfectant with a fungal claim ${ }^{9}$ should be used to perform a terminal clean once the patient has left the isolation room. Screening of patients for colonisation has been recommended in high-income countries with sporadic or no documented cases. However, this may not be feasible or appropriate in South Africa.

The emergence of $C$. auris as an important cause of nosocomial infection in South Africa will require swift changes to laboratory, clinical management and IPC algorithms. Local epidemiological studies to determine the relative importance of $C$. auris as a cause of healthcare-associated infections, risk factors for $C$. auris fungaemia and the niche of this pathogen in the hospital environment are currently underway.

\section{References}

1. Satoh K, Makimura K, Hasumi $Y$, et al. Candida auris sp. nov., a novel ascomycetous yeast isolated from the external ear canal of an inpatient in a Japanese hospital. Microbiol Immunol. 2009;53(1):41-4. http://dx.doi.org/10.1111/mim.2009.53.issue-1

2. Etienne $\mathrm{K}$, Vallabhaneni S, Farooqi J, et al. Epidemiology and whole genome sequence typing of globally emerging, multidrug-resistant Candida auris. Abstract for presentation at: ID Week 2016, Oct; USA.

3. Kim M-N, Shin JH, Sung H, et al. Candida haemulonii and closely related species at 5 university hospitals in Korea: Identification, antifungal susceptibility, and clinical features. Clin Infect Dis. 2009;48(6):e57-e61. http://dx.doi.org/10.1086/598174
4. Chowdhary A, Anil Kumar VA, Sharma C, et al. Multidrugresistant endemic clonal strain of Candida auris in India. Eur J Clin Microbiol Infect Dis. 2014;33(6):919-26. http://dx.doi.org/10.1007/s10096-013-2027-1

5. Chowdhary A, Sharma C, Duggal S, et al. New clonal strain of Candida auris, Delhi, India. India. Emerg Infect Dis. 2013;19(10):1670-3. http://dx.doi.org/10.3201/eid1910.130393

6. Lee WG, Shin JH, Uh Y, et al. First three reported cases of nosocomial fungemiacausedbyCandidaauris.JClinMicrobiol.2011;49(9):3139-42. http://dx.doi.org/10.1128/JCM.00319-11

7. Emara M, Ahmad S, Khan Z, et al. Candida auris candidemia in Kuwait, 2014. Emerg Infect Dis. 2015;21(6):1091-2. http://dx.doi.org/10.3201/eid2106.150270

8. Magobo RE, Corcoran C, Seetharam S, et al. Candida auris-associated candidemia, South Africa. Emerg Infect Dis. 2014;20:1250-1.

9. Centers for Disease Control and Prevention. Clinical alert to U.S. healthcare facilities: Global emergence of invasive infections caused by the multidrug-resistant yeast Candida auris 2016. Available from: http://www.cdc.gov/fungal/diseases/candidiasis/candida-aurisalert.html.

10. Prakash A, Sharma C, Singh A et al. Evidence of genotypic diversity among Candida auris isolates by multilocus sequence typing, matrixassisted laser desorption ionization time-of-flight mass spectrometry and amplified fragment length polymorphism. Clin Microbiol Infect. 2016;22(3):277, e1-e9.

11. Magobo RE, Corcoran C, Govender NP. Molecular typing of multidrugresistant Candida auris strains in Gauteng province, South Africa. Abstract submitted to: ASLM; 2016, Dec; South Africa.

12. Public Health England. Guidance for the laboratory investigation, management and infection prevention and control for cases of Candida auris 2016. Available from: www.gov.uk/government/ collections/candida-auris.

Received: 01-08-2016 Accepted: 04-08-2016 